\title{
Remote Ischemic Preconditioning of the Femoral Artery and Vein does not Protect against Renal Ischemia/Reperfusion-Induced Injury in Anesthetized Mice
}

\author{
Yifan Zhang ${ }^{1}$, Daisuke Nakano ${ }^{2 *}$, Akira Nishiyama ${ }^{2}$ \\ ${ }^{1}$ Department of No.2 Orthopedics, Shijiazhuang City No.1 Hospital, Shijiazhuang, Hebei, China \\ ${ }^{2}$ Department of Pharmacology, Kagawa University Medical School, Kagawa, Japan
}

Received: April 23, 2016; Accepted: May 13, 2016; Published: May 20, 2016

*Corresponding author: Daisuke Nakano, Ph.D., Department of Pharmacology, Faculty of Medicine, Kagawa University, $1750-1$ Ikenobe, Miki, Kita, Kagawa 761-0793, Japan, Tel: +81 (87) 891-2125; Fax: +81 (87) 891-2126; E-mail: dnakano@med.kagawa-u.ac.jp

\begin{abstract}
Multiple cycles of short Ischemia/Reperfusion (I/R) of the $\operatorname{limb}(\mathrm{s})$ is hypothesized to be a potential therapeutic strategy against I/R-induced injury of the peripheral organs, so-called Remote Ischemic Preconditioning (RIPC). Our aim is to examine the efficacy of experimental RIPC for renal I/R-induced injury in mice for the future further analysis. We investigated the effects of seven different RIPC protocols involving the right femoral artery and vein of either isoflurane- or pentobarbital-anesthetized mice. However, none of these protocols protected the kidney against I/R injury. In conclusion, RIPC of a direct clamping of femoral artery and vein gave no protection against renal I/R-induced injury in anesthetized mice.
\end{abstract}

Keywords: Ischemia reperfusion; Remote ischemic preconditioning; Anesthesia

\section{Introduction}

Acute Kidney Injury (AKI), which is diagnosed, based on oliguria and/or a decrease in glomerular filtration rate, is associated with high morbidity and mortality, and is one of the most common complications of major cardiovascular surgery, percutaneous coronary intervention and kidney transplantation [1]. The current therapeutic approaches against AKI are to maintain systemic hemodynamic and fluid balance and dialysis. However, the incidence and mortality rates remain high.

We previously reported that Renal Ischemic Preconditioning (IPC), which consisted of multiple cycles of short ischemia and reperfusion of the renal artery and vein, created a resistance to renal ischemia/reperfusion injury [2]. An experimental limitation of that study was that IPC of the renal artery and vein is not a realistic procedure in a clinical setting. To overcome this limitation, there is increasing interest in Remote IPC (RIPC), IPC performed at a site remote to the target organs, as an effective prophylactic strategy against ischemic organ damage. However, the outcomes of clinical studies examining the benefits of RIPC are not consistent; some reported benefits [3-5], while others reported neutral effects $[6,7]$. Therefore, the aim of the present study is to develop an experimental RIPC model against Renal Ischemia/Reperfusion (I/R)-induced AKI for the further evaluation of complexity observed in the clinical studies.

\section{Methods and Materials}

Male C57BL/ 6 mice at 5 weeks of age were purchased from CLEA (Tokyo, Japan). The mice were uninephrectomized 10 days prior to I/R and RIPC. We examined seven different RIPC protocols to determine whether RIPC induces renal protection against renal I/R injury in either pentobarbital (Figure 1 and 2) or isoflurane (Figure 3) anesthetized mice. All experimental procedures were performed according to the guidelines for the care and use of animals established by Kagawa University.

RIPC was conducted by occluding the femoral artery and vein in the right hind limb by noninvasive vascular clamp, except for one protocol using low temperature-induced ischemia. We observed the right hind limb turn white during ischemia, and return to normal color after reperfusion. Renal I/R were performed in the left kidney of animals that had received a right uninephrectomized 10 days prior to $I / R$, under body temperature-controlled condition. Blood Urea Nitrogen (BUN) level was assessed as previously described [2]. Plasma BUN levels were measured using commercially available assay kits (Urea nitrogen B test; Wako, Osaka, Japan).

For the histology, kidney tissue was fixed with 4\% par formaldehyde $(\mathrm{pH}=7.4)$, embedding in paraffin and sectioned into $3 \mu \mathrm{m}$ thick slides, and stained with hematoxylin/ eosin. We used an optical microscope (BX-51/DP-72; Olympus, Tokyo, Japan) to observe renal histological changes. Semi-quantitatively analyzed HE scores were given as average values of loss of the brush border, tubular dilatation, cast formation, and congestion/ hemorrhage. 

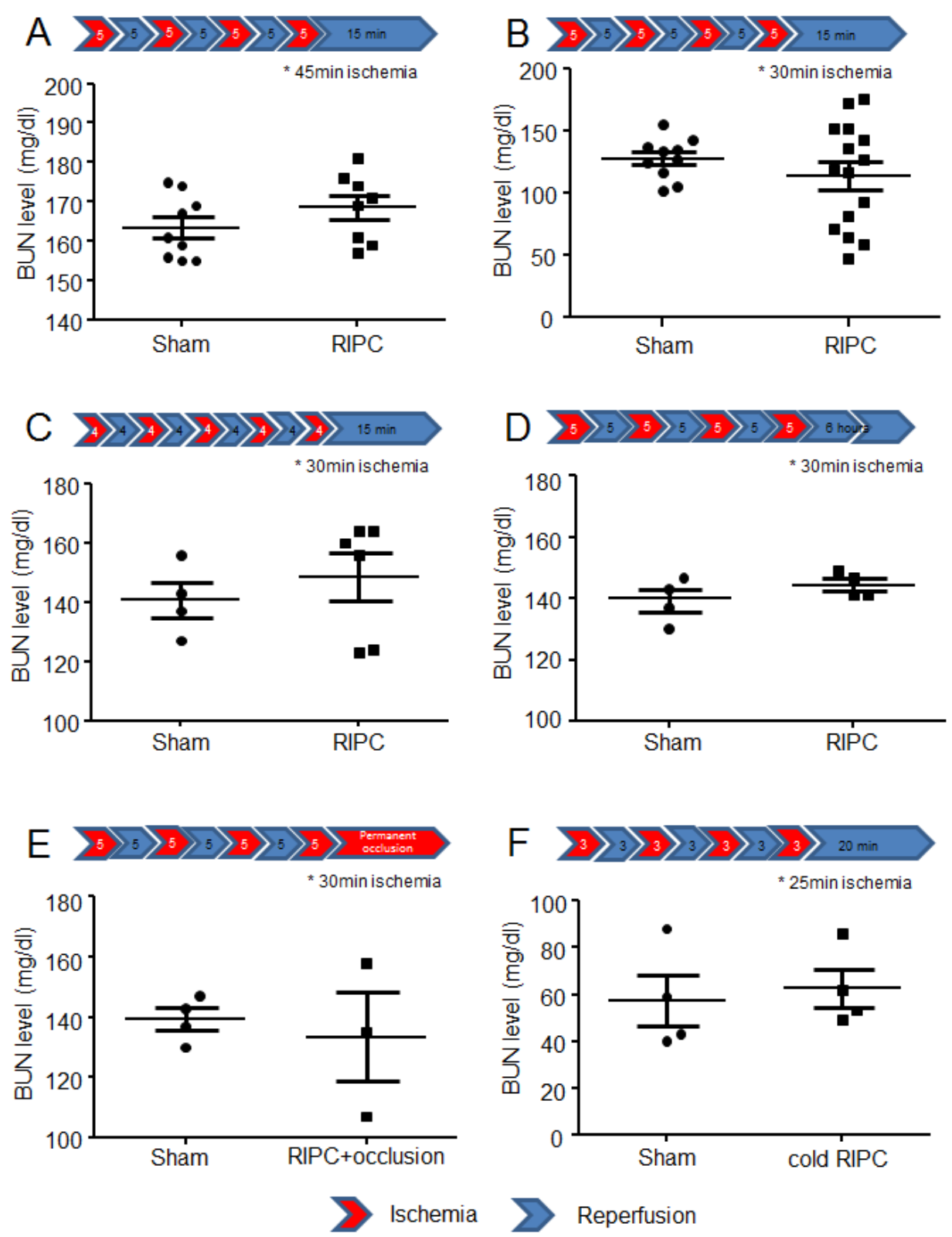

Figure 1: Effects of RIPC on BUN levels at 24 hours after I/R injury. RIPC was performed as indicated in each graph. Mice received 45min (A), 30min (B-E) and $25 \mathrm{~min}$ (F) ischemia followed by reperfusion, after RIPC. The difference in RIPC protocol was depicted in the arrows in each figure. Plasma BUN levels at 24 hours post reperfusion showed no significant difference between the sham and RIPC groups.

Results are expressed as means \pm standard error of the mean. Statistical significance was assessed using one-way ANOVA followed by Turkey's multiple comparison tests using the program Graph Pad Prism 5. Student's $t$ tests were performed to compare the means in experiments with two individual groups. Values of $P<0.05$ were considered statistically significant.

\section{Results}

RIPC consisting of four cycles of $5 \mathrm{~min}$ ischemia followed by $5 \mathrm{~min}$ reperfusion before I/R, did not attenuate BUN levels at 24 hours after $45 \mathrm{~min}$ ischemia and the following reperfusion (Figure
1A). Similar morphological changes in the kidney, including cast formation and tubular necrosis, were observed between sham and RIPC groups (Figure 2A and 2B).

The severe AKI induced after $45 \mathrm{~min}$ ischemia may halt the mechanism of reno-protection induced by RIPC. Thus, we investigated a milder I/R injury with $30 \mathrm{~min}$ ischemia (Figure 1BF). RIPC still did not show Reno-protective effects in this milder I/R model (Figure 1B). Compared with $45 \mathrm{~min}$ I/R injury, $30 \mathrm{~min}$ I/R injury induced medium cast formation and tubular necrosis; however there was no difference in severity between sham and RIPC groups (Figure 2C and 2D). We next changed the RIPC 


\section{A: Sham + IR 45-min}

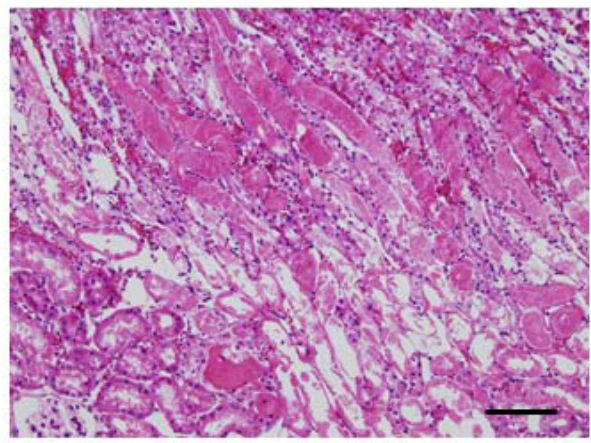

C: Sham + IR-30min

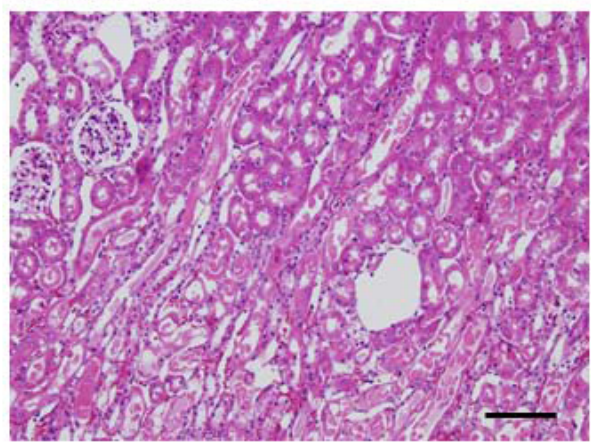

\section{B: RIPC + IR 45-min}

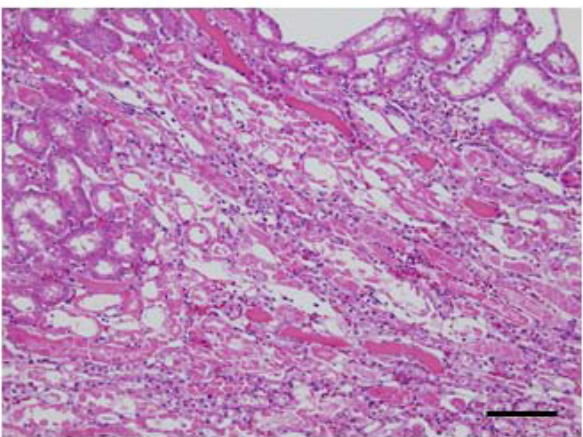

$\mathrm{D}: \mathrm{RIPC}+\mathrm{IR}$ 30-min

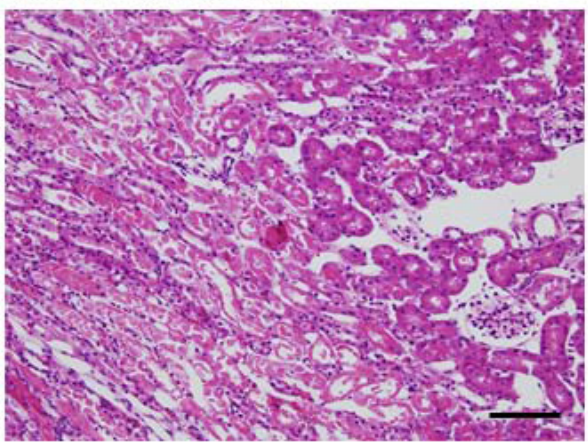

Figure 2: Effects of RIPC on histological injury at 24 hours after I/R injury. The images were taken in the kidneys that received RIPC consisting of four cycles of $5 \mathrm{~min}$ ischemia followed by $5 \mathrm{~min}$ reperfusion before either 45 -min (upper images) or $30 \mathrm{~min}$ (bottom images) of ischemia. There is no significant difference in the histological damage between the groups. Images are from representative mice treated with protocol A or B. Scale bars: $200 \mu \mathrm{m}$.

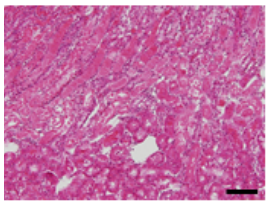

Sham

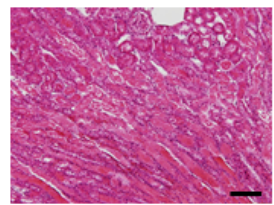

RIPC

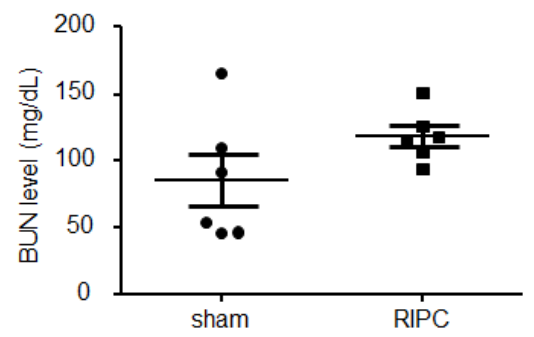

Figure 3: Effects of RIPC on acute kidney injury after I/R injury in isoflurane-anesthetized mice. The mice received RIPC consisting of four cycles of 5 $\mathrm{min}$ ischemia followed by $5 \mathrm{~min}$ reperfusion before 30 -min of ischemia and $24 \mathrm{~h}$ reperfusion. RIPC did not improve I/R-induced kidney injury.

protocol from $5 \mathrm{~min}$ ischemia/5min reperfusion for 4 cycles to $4 \mathrm{~min}$ ischemia/4min reperfusion for 6 cycles (Figure 1C). Again, RIPC did not significantly affect I/R-induced injury (Figure 1C).

The duration of the recovery time after RIPC prior to I/R was for $15 \mathrm{~min}$ in the experimental protocol above. Therefore, the recovery time was prolonged from $15 \mathrm{~min}$ to $6 \mathrm{hr}$ (Figure 1D).
The extension of recovery time did not improve the effect of RIPC in I/R-induced injury. We additionally examined the severity of renal injury in mice with a permanently occluded femoral artery after RIPC. I/R performed $6 \mathrm{hr}$ after RIPC increased BUN similarly in the mice that received a sham-RIPC with permanent occlusion compared with the mice that underwent recovery without permanent occlusion (Figure 1E). 
Another set of animals received RIPC using an ice pack instead of clamping the femoral artery and vein. The decreased blood flow in the hind limb was confirmed by the color of the then are, and the reperfusion during preconditioning was supported by a hand warmer. The ice-induced preconditioning showed no effects on BUN levels at $24 \mathrm{hr}$ compared with BUN levels in the sham group (Figure 1F).

There are reports indicating that anesthetics influence the effects of RIPC [8]. We therefore changed anesthetics from pentobarbital to isoflurane, an inhalation anesthetic. Isoflurane administration was stopped immediately after reperfusion and suturing of the surgical field. RIPC in isoflurane-anesthetized mice did not alleviate the I/R-induced injury (Figure 3).

\section{Discussion}

We failed to observe any beneficial effects against I/Rinduced AKI of several RIPC protocols in either pentobarbital- or isoflurane-anesthetized uninephrectomized C57/Bl6J mice. A limitation of the present study is that the mice were anesthetized. The anesthetics used might interfere or mask the mechanism of RIPC-induced peripheral organ protection if it is through a central nervous system pathway [9-11]. Indeed, a previous study showed that remote ischemic preconditioning protects the kidney against AKI by suppressing renal sympathetic nerve activity in Sprague Dawley rats [9]. However, two recent clinical trials reported that RIPC did not improve clinical outcomes in patients undergoing cardiac surgery [12], or coronary-artery bypass graft surgery [13]. The former study was performed under total anesthesia with intravenous protocol, which might interfere with RIPC effects [8], while the later study was done under nonstandardized anesthetics. Therefore, anesthetics themselves, not a specific anesthetic drug, might limit the effects of RIPC. We did not perform RIPC in conscious mice as that procedure imparts an even more severe influence on their nervous system than using only anesthetics.

There might be difference on the sensitivity to RIPC between the species. RIPC to the hind-limb of rats has reported to demonstrate the protective effects $[11,14,15]$. On the other hand, to our knowledge, there is no report showing the protective effect of RIPC against AKI in mice, and the current study showed that mice were insensitive to the multiple protocols of RIPC. Since we previously showed that the marine kidney is sensitive to the ischemic preconditioning procedure on renal pedicle against I/Rinduced AKI [2], the species difference between rats and mice may result from the extra-renal mechanism.

The other possibility for why RIPC did not show its protective effect in the present study unlike the other reports $[14,15]$, might be due to the detailed surgical procedure. We dissected the femoral artery and vein from surrounding tissues to induce complete ischemia by a clamp; however, most of study only ties the hind limb. The latter case could give stress on the skeletal muscle and might stimulate the interaction between muscle and the kidney $[16,17]$.

Although we did not find any benefit of RIPC in animals under anesthesia, a common finding among both basic and clinical studies is that RIPC did not exaggerate or increase the risk of AKI. RIPC is thus still worth considering as a potential choice of prophylactic treatment against AKI because of its non-drug and non-invasive nature. For this purpose, the RIPC protocol that can induce the benefits must be discussed and shared widely, for future patients who might potentially receive benefits from this procedure.

\section{Acknowledgements}

This research is supported by the fund for Kagawa University Young Scientists 2014.

\section{References}

1. Best PJ, Lennon R, Ting HH, Bell MR, Rihal CS, Holmes DR, et al. The impact of renal insufficiency on clinical outcomes in patients undergoing percutaneous coronary interventions. J Am Coll Cardiol. 2002;39(7):1113-9.

2. Nishioka S, Nakano D, Kitada K, Sofue T, Ohsaki H, Moriwaki K, et al. The cyclin-dependent kinase inhibitor p21 is essential for the beneficial effects of renal ischemic preconditioning on renal ischemia/reperfusion injury in mice. Kidney Int. 2014;85(4):871-9. doi:10.1038/ki.2013.496

3. Zimmerman RF, Ezeanuna PU, Kane JC, Cleland CD, Kempananjappa TJ, Lucas FL, et al. Ischemic preconditioning at a remote site prevents acute kidney injury in patients following cardiac surgery. Kidney Int. 2011;80(8):861-7. doi: 10.1038/ki.2011.156.

4. Veighey K, MacAllister R. Clinical applications of remote ischaemic preconditioning in native and transplant acute kidney injury. Pediatr Nephrol. 2015;30(10):1749-59. doi: 10.1007/s00467-014-2965-6.

5. Er F, Nia AM, Dopp H, Hellmich M, Dahlem KM, Caglayan E, et al. Ischemic preconditioning for prevention of contrast mediuminduced nephropathy: randomized pilot RenPro Trial (Renal Protection Trial). Circulation. 2012;126(3):296-303.doi: 10.1161/ CIRCULATIONAHA.112.096370.

6. Gallagher SM, Jones DA, Kapur A, Wragg A, Harwood SM, Mathur R, et al. Remote ischemic preconditioning has a neutral effect on the incidence of kidney injury after coronary artery bypass graft surgery. Kidney Int. 2015;87(2):473-81. Epub 2014/07/31. doi: 10.1038/ ki.2014.259.

7. Sterenborg TB, Menting TP, de Waal Y, Donders R, Wever KE, Lemson $\mathrm{MS}$, et al. Remote ischemic preconditioning to reduce contrast-induced nephropathy: study protocol for a randomized controlled trial. Trials. 2014;15:119. doi: 10.1186/1745-6215-15-119.

8. Kottenberg E, Thielmann M, Bergmann L, Heine T, Jakob H, Heusch G, et al. Protection by remote ischemic preconditioning during coronary artery bypass graft surgery with isoflurane but not propofol - a clinical trial. Acta Anaesthesiol Scand. 2012;56(1):30-8. doi: 10.1111/j.13996576.2011.02585.x.

9. Oba T, Yasukawa H, Nagata T, Kyogoku S, Minami T, Nishihara $\mathrm{M}$, et al. Renal Nerve-Mediated Erythropoietin Release Confers Cardioprotection During Remote Ischemic Preconditioning. Circ J. 2015;79(7):1557-67. doi: 10.1253/circj.CJ-14-1171.

10. Mastitskaya S, Marina N, Gourine A, Gilbey MP, Spyer KM, Teschemacher AG, et al. Cardioprotection evoked by remote ischaemic preconditioning is critically dependent on the activity of vagal pre-ganglionic neurones. Cardiovasc Res. 2012;95(4):487-94. doi: $10.1093 /$ cvr/cvs212.

11.Wong GT, Lu Y, Mei B, Xia Z, Irwin MG. et al. Cardioprotection from 
remote preconditioning involves spinal opioid receptor activation. Life Sci. 2012;91(17-18):860-5. doi: 10.1016/j.lfs.2012.08.037.

12. Meybohm P, Bein B, Brosteanu O, Cremer J, Gruenewald M, Stoppe C, et al. A Multicenter Trial of Remote Ischemic Preconditioning for Heart Surgery. N Engl J Med. 2015 Oct 8;373(15):1397-407. doi: 10.1056/NEJMoa1413579.

13. Hausenloy DJ, Candilio L, Evans R, Ariti C, Jenkins DP, Kolvekar S, et al. Remote Ischemic Preconditioning and Outcomes of Cardiac Surgery. N Engl J Med. 2015;373(15):1408-17. doi: 10.1056/NEJMoa1413534.

14. Sedaghat Z, Kadkhodaee M, Seifi B, Salehi E, Najafi A, Dargahi L, et al. Remote preconditioning reduces oxidative stress, downregulates cyclo-oxygenase-2 expression and attenuates ischaemia-reperfusioninduced acute kidney injury. Clin Exp Pharmacol Physiol. 2013;40(2):97-103. doi: 10.1111/1440-1681.12044.
15.Wever KE, Masereeuw R, Wagener FA, Verweij VG, Peters JG, Pertijs JC, et al. Humoral signalling compounds in remote ischaemic preconditioning of the kidney, a role for the opioid receptor. Nephrol Dial Transplant. 2013;28(7):1721-32. doi: 10.1093/ndt/gfs601.

16. McIntire KL, Chen Y, Sood S, Rabkin R. Acute uremia suppresses leucine-induced signal transduction in skeletal muscle. Kidney Int 2014;85(2):374-82. doi: 10.1038/ki.2013.216.

17. Pavyde E, Maciulaitis R, Mauricas M, Sudzius G, Ivanauskaite Didziokiene E, Laurinavicius A, et al. Skeletal Muscle-Derived Stem/Progenitor Cells: A Potential Strategy for the Treatment of Acute Kidney Injury. Stem Cells Int. 2016;2016:9618480. doi: $10.1155 / 2016 / 9618480$. 